\title{
Напряженные многослойные структуры с псевдоморфными слоями GeSiSn
}

\author{
(C) В.А. Тимофеев ${ }^{+}$, А.И. Никифоров ${ }^{+*}$, А.Р. Туктамышев ${ }^{+}$, М.Ю. Есин ${ }^{+}$, В.И. Машанов ${ }^{+}$, \\ А.К. Гутаковский ${ }^{+}$, Н.А. Байдакова
}

+ Институт фризики полупроводников им. А.В. Ржанова Сибирского отделения Российской академии наук, 630090 Новосибирск, Россия

* Национальный исследовательский Томский государственный университет,

634050 Томск, Россия

^ Институт физики микроструктур Российской академии наук, 607680 Нижний Новгород, Россия

E-mail: Vyacheslav.t@isp.nsc.ru

(Получена 27 апреля 2016 г. Принята к печати 10 мая 2016 г.)

Изучены температурные и композиционные зависимости критической толщины 2D-3D перехода для пленки GeSiSn на поверхности $\mathrm{Si}(100)$. Впервые исследованы закономерности формирования многослойных структур, содержащих псевдоморфные слои GeSiSn непосредственно на $\mathrm{Si}$ без релаксированных буферных слоев. Методом просвечивающей электронной микроскопии показана возможность создания многослойных структур на основе псевдоморфных пленок GeSiSn и определены параметры кристаллической решетки. Выращенные структуры продемонстрировали фотолюминесценцию для содержания Sn в слоях GeSiSn от 3.5 до $5 \%$.

\section{1. Введение}

В последние годы соединения на основе материалов $\mathrm{Ge}-\mathrm{Si}-\mathrm{Sn}$ привлекли к себе особое внимание в связи с возможностью их применения в интегральной кремниевой фотонике, микро- и наноэлектронике, фотовольтаике, термофотовольтаике, а также в области телекоммуникаций и инфракрасного обнаружения [1-6]. При добавлении $\mathrm{Sn}$ в $\mathrm{Ge}$ становится возможным управление постоянной решетки, напряжением, энергетической диаграммой, подвижностью носителей, эффективной массой носителей заряда и дефектами. Вдобавок, минимум зоны проводимости для L- и Г-долины уменьшается с увеличением содержания Sn, причем, уменьшение в Г-точке происходит быстрее. В результате, $\mathrm{GeSn}$ может стать прямозонным полупроводником при содержании $\mathrm{Sn} 10 \%$ для релаксированных слоев и $6 \%$ для пленок с деформацией растяжения $[7,8]$. Успехи в области роста эпитаксиальных слоев $\mathrm{GeSn}, \mathrm{GeSiSn}$ [9-11] открывают путь в направлении модификации зонной структуры путем управления напряжениями и составом. Помимо изменения электронных и оптических свойств, присутствие $\mathrm{Sn}$ на поверхности увеличивает поверхностную диффузию адатомов [12], а также влияет на появление серии сверхструктур, которые не наблюдаются в системе $\mathrm{GeSi}$ [13]. Основные проблемы синтеза эпитаксиальных пленок GeSn и GeSiSn, связанные с низкой равновесной растворимостью $\mathrm{Sn}$ в $\mathrm{Ge}$ и $\mathrm{Si}(<1 \%)$, сегрегацией и преципитацией, решаются с помощью неравновесных методик роста, таких как молекулярно-лучевая эпитаксия (МЛЭ), магнетронное распыление, твердофазная эпитаксия, перекристаллизация и газофазная эпитаксия (ГФЭ) [14-16]. Эпитаксиальные слои на основе материалов $\mathrm{Ge}-\mathrm{Si}-\mathrm{Sn}$ получены вплоть до $25 \% \mathrm{Sn}$ путем понижения температуры роста, контроля несоответствия решеток и напряженного состояния [17]. В большинстве работ, посвященных росту соединений GeSn, GeSiSn, демонстрируются структуры с толстыми релаксированными слоями Ge, GeSn или GeSiSn. Главными недостатками таких структур являются прорастающие дислокации, ухудшающие структурные и оптические свойства материала.

В представленной статье предлагается использовать взамен релаксированных слоев псевдоморфные упругонапряженные пленки GeSiSn, выращенные прямо на Si. Основное преимущество псевдоморфных пленок над толстыми слоями заключается в том, что они не содержат дислокаций и когерентны с подложкой. Сами пленки $\mathrm{GeSiSn}$ термически стабильнее в сравнении с GeSn [18] и имеют независимую регулировку параметра решетки и ширины запрещенной зоны. Нами получены напряженные многослойные структуры с псевдоморфными слоями $\mathrm{GeSiSn}$, демонстрирующие фотолюминесценцию для содержания $\mathrm{Sn}$ в слоях GeSiSn от 3.5 до $5 \%$.

\section{2. Эксперимент}

Методом молекулярно-лучевой эпитаксии в условиях сверхвысокого вакуума были получены многослойные структуры, содержащие псевдоморфные слои $\mathrm{GeSiSn}$ различного состава (содержание $\mathrm{Sn}$ от 0 до 10\%) и толщины (от 2 до 3.5 нм). Температура и скорость роста слоев GeSiSn, в многослойной структуре, варьировалась в диапазоне $100-150^{\circ} \mathrm{C}$ и $0.075-0.43 \mathrm{MC} / \mathrm{c}$ (1MC $\mathrm{Sn}$ на поверхности $\mathrm{Si}(100)$ равен $0.184 \mathrm{HM})$ соответственно. Слои GeSiSn заращивались $\mathrm{Si}$ при температуре $500^{\circ} \mathrm{C}$. Изменение морфологии и структуры поверхности при росте пленок GeSiSn и $\mathrm{Si}$ контролировалось с помощью дифракции быстрых электронов (ДБЭ). Энергия 
электронов составляла 20 кэВ. Анализ пространственновременных распределений интенсивности картин ДБЭ позволил идентифицировать сверхструктуры и начало формирования островков. Момент перехода 2D-3D определялся по временной зависимости интенсивности картины ДБЭ вдоль одного из тяжей, в направлении которого появляется объемный рефлекс. На основе методики определения 2D-3D перехода строилась кинетическая диаграмма роста пленок GeSiSn на $\mathrm{Si}(100)$ в диапазоне температур $150-500^{\circ} \mathrm{C}$. Исходя из кинетической диаграммы, задавалась толщина псевдоморфного слоя GeSiSn в многослойной структуре. Шероховатость поверхности пленок с многослойной структурой, содержащей гетеропереходы $\mathrm{GeSiSn} / \mathrm{Si}$, измерялась с помощью атомно-силовой микроскопии. Кристаллическая структура растущих слоев изучалась, используя поперечную высокоразрешающую просвечивающую электронную микроскопию (ПЭМ) с электронным микроскопом JEOL-4000EX (энергия электронов - 400 кэВ, разрешение - 0.165 нм). Количественная обработка изображений выполнена с помощью программного обеспечения цифровых микрофотографий (GATAN). Ионное травление использовалось для подготовки поперечных сечений образцов. Визуализация и количественное измерение решеточных искажений и деформационных полей выполнено с помощью метода геометрической фазы [19]. Оптические свойства структур исследовались методом спектроскопии фотолюминесценции (ФЛ), используя монохроматор ACTON 2300i и охлаждаемый детектор OMA-V на основе линейки InGaAs-фотодиодов с полосой чувствительности от 1.1 до 2.2 мкм. Для возбуждения фотолюминесценции использовалось излучение лазера $\mathrm{Nd}$ : YAG (длина волны 532 нм).

\section{3. Результаты и обсуждение}

Получение многослойных структур, содержащих упругонапряженные псевдоморфные слои GeSiSn, потребовало изучения температурных и композиционных зависимостей критической толщины $2 \mathrm{D}-3 \mathrm{D}$ перехода для пленки GeSiSn при различных несоответствиях решетки. Несоответствие параметров решеток GeSiSn и $\mathrm{Si}$ варьировалось вплоть до 5\%. На рис. 1 представлена кинетическая диаграмма роста GeSiSn при несоответствии 1 и $2 \%$. При повышении концентрации $\mathrm{Sn}$ для несоответствия $2 \%$ практически во всем диапазоне температур $150-450^{\circ} \mathrm{C}$ наблюдается увеличение критической толщины $2 \mathrm{D}-3 \mathrm{D}$ перехода, что наблюдалось для несоответствия параметров решеток $\mathrm{GeSiSn}$ и $\mathrm{Si}$, равного $4 \%$ и соединений GeSn [13]. На кривой 2 (рис. 1) для состава $\mathrm{Ge}_{0.315} \mathrm{Si}_{0.65} \mathrm{Sn}_{0.035}$ отчетливо видны два экстремума вблизи 250 и $325^{\circ} \mathrm{C}$. В области между первым и вторым экстремумами происходит изменение сверхструктуры, что снижает напряжения, накопленные в пленке, и критическая толщина 2D-3D перехода растет. Выше $325^{\circ} \mathrm{C}$ вследствие сегрегации [20] олово собирается на поверхности и при $450^{\circ} \mathrm{C}$ наблюдается $2 \mathrm{D}-3 \mathrm{D}$

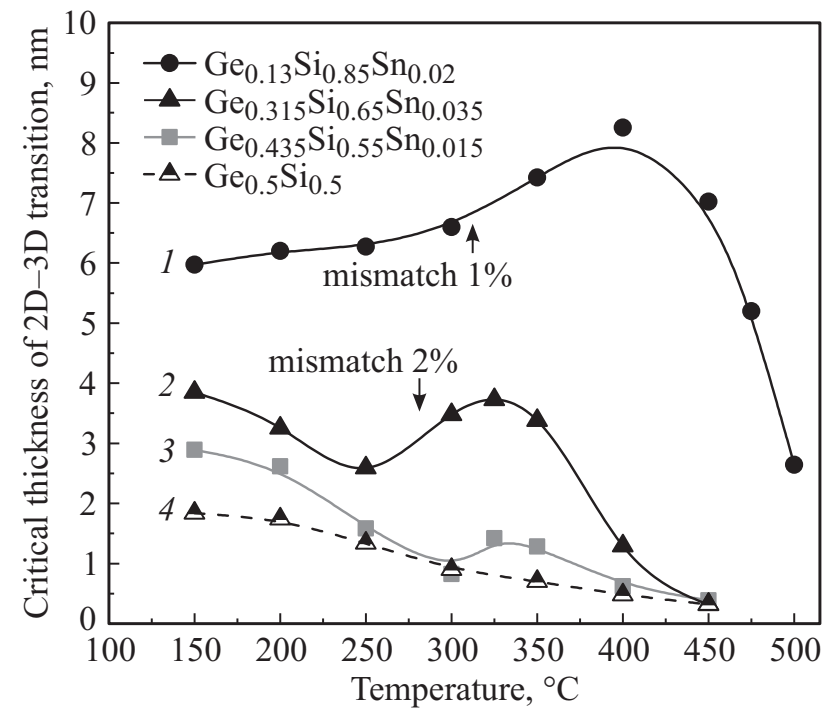

Рис. 1. Зависимости критической толщины $2 \mathrm{D}-3 \mathrm{D}$ перехода для пленок GeSiSn. Кривая $1-$ несоответствие параметров решеток $\mathrm{GeSiSn}$ и $\mathrm{Si}$ составляет $1 \%$; кривые $2-4-$ несоответствие $2 \%$.

переход для слоя по составу близкому к двойному соединению со сниженной концентрацией $\mathrm{Sn}$. Сегрегация $\mathrm{Sn}$ на поверхность пленки зависит от температуры роста, от содержания Sn, а также от несоответствия параметров решеток GeSiSn и Si. Уменьшение содержания $\mathrm{Sn}$ в тройном соединении увеличивает пороговое значение температуры осаждения, при которой начинается сегрегация Sn. Температура роста GeSiSn выбиралась такой, чтобы оставаться в условиях эпитаксиального роста и подавить эффект сегрегации Sn. Оптимальный температурный диапазон осаждения GeSiSn занимает область от 100 до $200^{\circ} \mathrm{C}$. На основе кинетических диаграмм роста определялась толщина псевдоморфной пленки GeSiSn, которая достигала 3.85 нм для несоответствия $2 \%$ (рис. 1 , кривая 2) при температуре $150^{\circ} \mathrm{C}$. Уменьшая несоответствие параметров решеток GeSiSn и $\mathrm{Si}$ до $1 \%$ (рис. 1 , кривая 1), толщина псевдоморфной пленки GeSiSn может достигать 6 нм в области низких температур. В течение роста GeSiSn сверхструктура $(2 \times 1)$, характерная для чистой поверхности $\mathrm{Si}$, исчезает. При этом на картине ДБЭ наблюдается сверхструктура $(2 \times N)$. Далее псевдоморфный слой GeSiSn заращивался $\mathrm{Si}$ при температуре $500^{\circ} \mathrm{C}$. Попытка снизить температуру роста $\mathrm{Si}$ ниже $450^{\circ} \mathrm{C}$ приводила к увеличению шероховатости поверхности, что фиксировалось как уширение рефлексов и увеличение диффузного фона на дифракционной картине. Толщина слоя $\mathrm{Si}$ при температуре $500^{\circ} \mathrm{C}$ выбиралась такой, чтобы получить гладкую поверхность, и изменялась в диапазоне от 5 до 20 нм в зависимости от состава пленки GeSiSn и содержания $\mathrm{Sn}$. В результате сегрегации олова на поверхность в процессе заращивания $\mathrm{GeSiSn}$ слоем $\mathrm{Si}$ наблюдается серия сверхструктур, сходных с теми, которые появляются при росте чистого олова субмонослойной толщины [21]. 


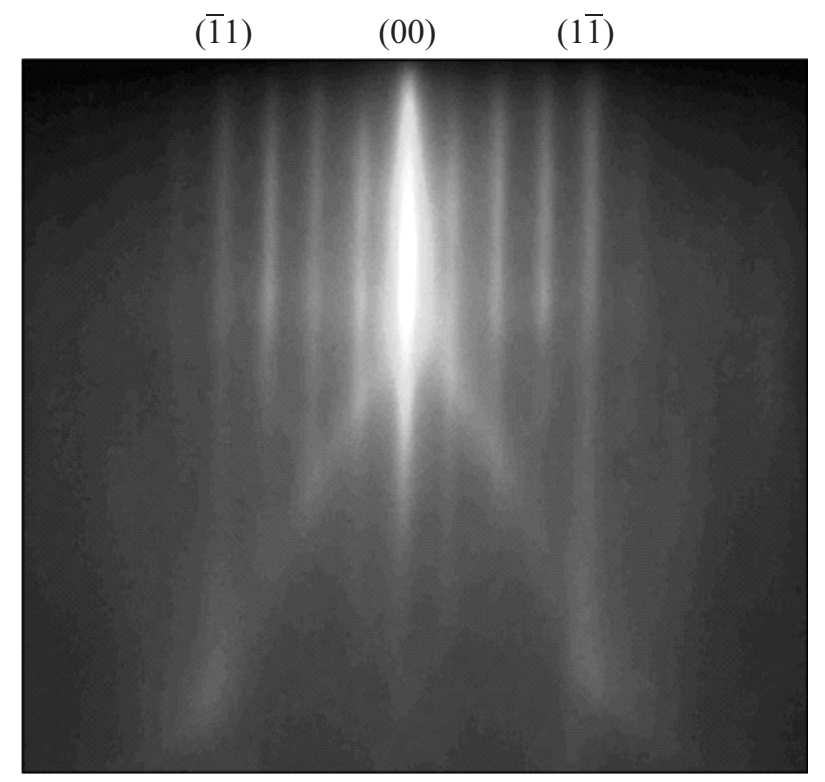

Рис. 2. Картина ДБЭ в направлении [100], наблюдаемая при росте $\mathrm{Si}$, покрывающего слой $\mathrm{GeSiSn}$ с содержанием $\mathrm{Ge} 31.5 \%$ и $\mathrm{Sn} 3.5 \%$ для несоответствия параметров решетки $\mathrm{GeSiSn}$ и $\mathrm{Si} 2 \%$.

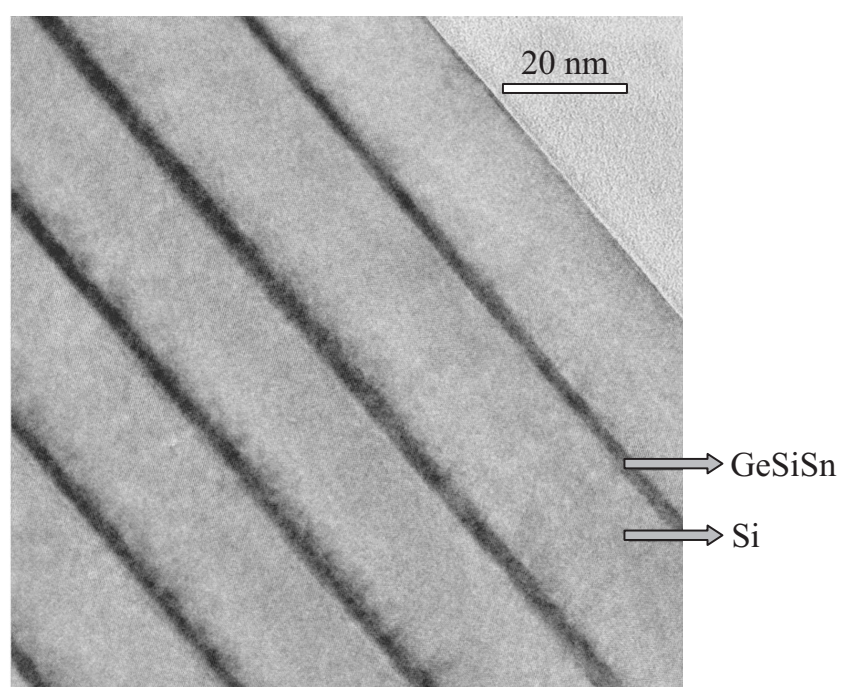

Рис. 3. Изображение ПЭМ поперечного среза многослойной структуры с гетеропереходами $\mathrm{Ge}_{0.5} \mathrm{Si}_{0.45} \mathrm{Sn}_{0.05} / \mathrm{Si}$ и периодом $25 \mathrm{Hм}$.

На рис. 2 показана картина ДБЭ в азимуте [100], наблюдаемая при росте пленки $\mathrm{Si}$, покрывающей слой GeSiSn. На поверхности пленки Si наблюдается сверхструктура $(4 \times 4)$. Эта сверхструктура фиксируется на картинах ДБЭ при осаждении $\mathrm{Si}$ на всех периодах многослойной гетероструктуры $\mathrm{GeSiSn} / \mathrm{Si}$ при содержании $\mathrm{Sn}$ от 3 до $10 \%$ и температуре заращивания $500^{\circ} \mathrm{C}$. Увеличение содержания $\mathrm{Sn}$ от 10\% и выше приводит к появлению двухдоменной сверхструктуры $(5 \times 1)$ при росте $\mathrm{Si}$, покрывающего слой GeSiSn. Сверхструктура
$(5 \times 1)$ говорит об усилении эффекта сегрегации $\mathrm{Sn}$ на поверхность, поскольку наблюдается при большем покрытии $\mathrm{Sn}$ на $\mathrm{Si}(100)$ [21]. Рост $\mathrm{Si}$ в две стадии, включающие осаждение $\mathrm{Si}$ толщиной несколько нанометров при температуре роста слоя GeSiSn и дальнейший рост $\mathrm{Si}$ при температуре $500^{\circ} \mathrm{C}$, позволил сдерживать сегрегацию Sn, что фиксировалось сохранением сверхструктуры $(2 \times 1)$, характерной для чистой поверхности Si. Среднеквадратичная шероховатость поверхности многослойной структуры, содержащей гетеропереходы $\mathrm{GeSiSn} / \mathrm{Si}$, измерена методом атомно-силовой микроскопии и составляет 0.15 нм. Кристаллическое совершенство выращенных гетероструктур изучено методом просвечивающей электронной микроскопии (ПЭМ) (рис. 3). Из данных ПЭМ можно заключить, что наши структуры не содержат дислокаций и являются кристаллически совершенными. Используя метод геометрической фазы, были визуализированы и измерены искажения кристаллической решетки и деформационные поля на ПЭМ изображениях многослойной структуры с гетеропереходами $\mathrm{Ge}_{0.5} \mathrm{Si}_{0.45} \mathrm{Sn}_{0.05} / \mathrm{Si}$. Экспериментально измеренные величины межплоскостных расстояний $\left(d_{002}=0.29 \mathrm{Hм}\right.$, $d_{111}=0.324 \mathrm{Hм}, d_{220}=0.192$ нм) для образца, представленного на рис. 3, однозначно соответствуют тетрагональной решетке с параметрами $a=0.543 \mathrm{Hм} \mathrm{и}$ $c=0.58$ нм. Отсюда следует, что слои $\mathrm{Ge}_{0.5} \mathrm{Si}_{0.45} \mathrm{Sn}_{0.05}$ имеют либо ненапряженную тетрагональную кристаллическую решетку, либо кубическую кристаллическую решетку, которая упруго деформирована и когерентно сопряжена на гетерогранице с кристаллической решеткой $\mathrm{Si}$, таким образом, имеет место формирование псевдоморфной пленки $\mathrm{Ge}_{0.5} \mathrm{Si}_{0.45} \mathrm{Sn}_{0.05}$. Для псевдоморфной пленки GeSiSn с кубической структурой, когда реализуется плосконапряженное состояние упругой деформации, $\varepsilon_{x x}$ и $\varepsilon_{y y}$ определяются из уравнений:

$$
\begin{gathered}
\varepsilon_{x x}=\left(a_{\mathrm{Si}}-a_{\mathrm{GeSiSn}}\right) / a_{\mathrm{GeSiSn}}, \\
\varepsilon_{y y}=-(2 v / 1-v) \varepsilon_{x x},
\end{gathered}
$$

где $a_{\mathrm{Si}}$ и $a_{\mathrm{GeSiSn}}-$ параметры решетки кремния и твердого раствора GeSiSn, v - коэффициент Пуассона. Кроме того, $\varepsilon_{y y}$ может быть вычислено также по формуле

$$
\varepsilon_{y y}=\left(c-a_{\mathrm{GeSiSn}}\right) / a_{\mathrm{GeSiSn}} .
$$

Подставляя (1) в (2) и приравнивая выражения (2) и (3), находим выражение для параметра кубической решетки GeSiSn:

$$
a_{\mathrm{GeSiSn}}=\frac{1-v}{1+v}\left(c+\frac{2 v}{1-v} a_{\mathrm{Si}}\right) .
$$

Для $v=0.3$ и $c=0.58$ нм параметр кубической решетки GeSiSn равен 0.562 нм. Полученное значение параметра решетки GeSiSn отличается от величины параметра решетки $\mathrm{Ge}_{0.5} \mathrm{Si}_{0.45} \mathrm{Sn}_{0.05}$, заданной условиями роста, на $0.5 \%$ (несоответствие параметров решетки $\mathrm{Ge}_{0.5} \mathrm{Si}_{0.45} \mathrm{Sn}_{0.05}$ и $\mathrm{Si}$ составляет 3\%). Зная параметр 


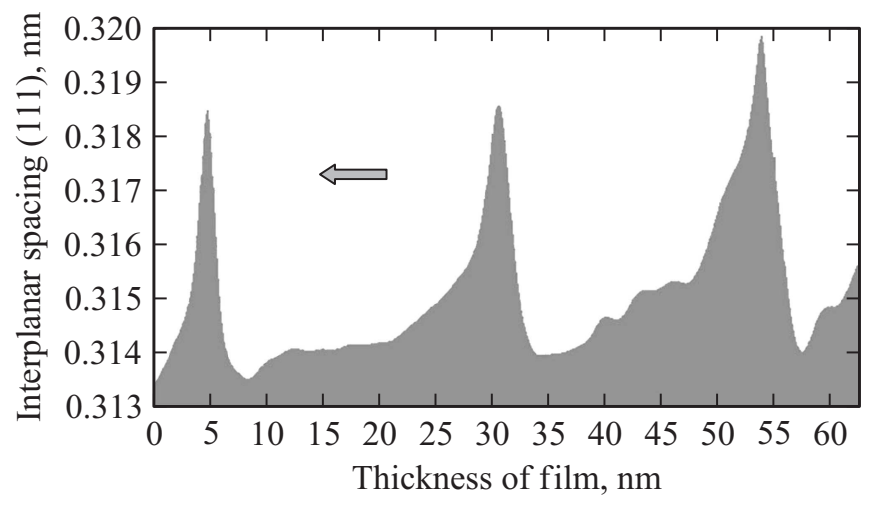

Рис. 4. Профиль межплоскостного расстояния (111) от толщины пленки, включающий несколько гетеропереходов $\mathrm{Ge}_{0.5} \mathrm{Si}_{0.45} \mathrm{Sn}_{0.05} / \mathrm{Si}$. Стрелкой указано направление к поверхности пленки.

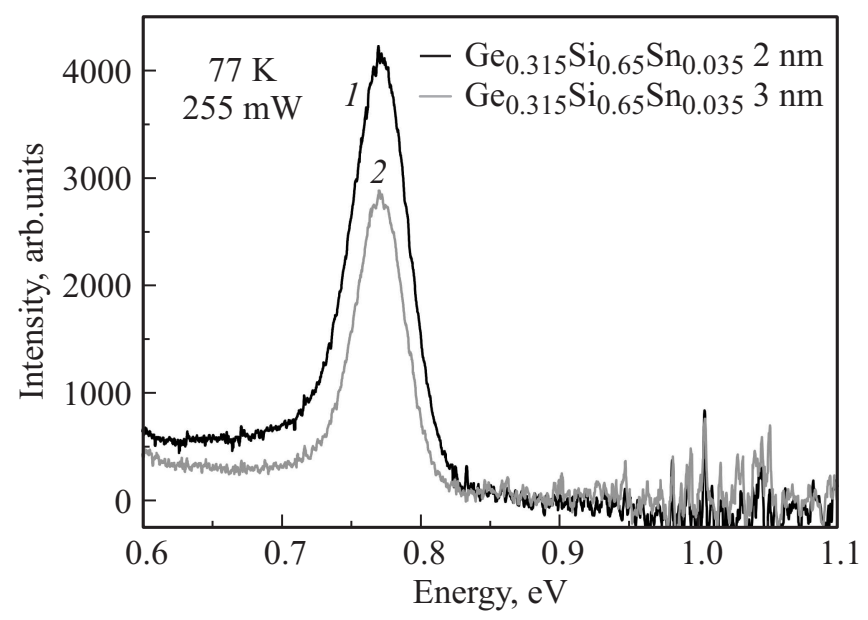

Рис. 5. Спектры фотолюминесценции от многослойных структур с псевдоморфными слоями $\mathrm{GeSiSn}$, имеющими толщину и температуру роста: 1) $2 \mathrm{HM}, 150^{\circ} \mathrm{C}$; 2) $3 \mathrm{HM}, 100^{\circ} \mathrm{C}$.

решетки GeSiSn, можно говорить о значении несоответствия этого параметра по отношению к параметру решетки $\mathrm{Si}$, однако о составе пленки ничего сказать нельзя, он задавался потоками $\mathrm{Ge}, \mathrm{Si}$ и $\mathrm{Sn}$. На рис. 4 продемонстрирован профиль изменения межплоскостного расстояния (111) от толщины пленки в направлении $[001]$ в прилегающей к ростовой поверхности области многослойной структуры, включающей слои $\mathrm{Ge}_{0.5} \mathrm{Si}_{0.45} \mathrm{Sn}_{0.05}$ и $\mathrm{Si}$. Во всех трех слоях GeSiSn на рис. 4 межплоскостное расстояние (111) имеет близкое значение и составляет примерно 0.32 нм. Анализ более удаленных от ростовой поверхности слоев, вплоть до прилегающего к подложке, дает те же самые значения. Хорошо наблюдаемое изменение межплоскостного расстояния (111) в слое $\mathrm{Si}$ вблизи GeSiSn (третий период от поверхности пленки) может быть вызвано сегрегацией $\mathrm{Sn}$. Как упоминалось выше, используя методику двухступенчатого роста $\mathrm{Si}$, удалось в значительной степени снизить влияние сегрегации в процессе заращивания $\mathrm{GeSiSn}$ слоем $\mathrm{Si}$, что подтверждается симметричными профилями межплоскостного расстояния от толщины пленки в области слоев тройных соединений GeSiSn.

Оптические свойства многослойных структур, включающих слои GeSiSn, изучены с помощью фотолюминесценции. Сигнал ФЛ возбуждался Nd:YAG лазером (532 нм), мощность накачки изменялась от 20 до 900 мВт. На рис. 5 приведены спектры фотолюминесценции, полученные при температуре $77 \mathrm{~K}$. Наблюдается люминесценция в диапазоне $0.7-0.85$ эВ с максимальной интенсивностью при энергии фотонов 0.77 эВ, что соответствует длине волны 1.61 мкм. С ростом толщины GeSiSn от 2 до 3 нм и уменьшением температуры роста от 150 до $100^{\circ} \mathrm{C}$ сигнал люминесценции снижается, что может быть вызвано увеличением дефектов в кристаллической структуре. В результате повышения содержания олова до $5 \%$ в слое GeSiSn люминесценция имеет место в диапазоне 0.6-1 эВ с максимальным значением 0.74 эВ (1.67 мкм). Продвижение в длинноволновую область более 2 мкм потребует увеличения содержания $\mathrm{Sn}$ в слоях GeSiSn до $10 \%$ и выше.

\section{4. Заключение}

Впервые исследованы закономерности формирования многослойных структур на квантовых ямах, содержащих псевдоморфные слои GeSiSn, не прибегая к использованию релаксированных буферных слоев, а создавая структуры прямо на Si. Данные ПЭМ подтвердили кристаллическое совершенство полученных образцов. Многослойные периодические структуры с гетеропереходами $\mathrm{GeSiSn} / \mathrm{Si}$ продемонстрировали фотолюминесценцию.

Работа выполнена при финансовой поддержке РФФИ (гранты № 14-29-07153, 16-32-60005, 16-32-00039, 16-29-03292). В статье использованы результаты, полученные в ходе выполнения проекта (№ 8.2.10.2015), в рамках Программы „Научный фонд им. Д.И. Менделеева Томского государственного университета“. Исследования ПЭМ были выполнены при поддержке РНФ (грант № 14-22-00143), используя оборудование центра коллективного пользования (ЦКП „Наноструктуры“).

\section{Список литературы}

[1] B. Vincent, Y. Shimura, S. Takeuchi, T. Nishimura, G. Eneman, A. Firrincieli, J. Demeulemeester, A. Vantomme, T. Clarysse, O. Nakatsuka, S. Zaima, J. Dekoster, M. Caymax, R. Loo. Microelect. Eng., 88, 342 (2011).

[2] B.R. Conley, H. Naseem, G. Sun, P. Sharps, Shui-Qing Yu. 38th IEEE Photovoltaic Specialists Conf. (Austin, Texas, 2012) p. 001189.

[3] M. Oehme, M. Schmid, M. Kaschel, M. Gollhofer, D. Widmann, E. Kasper, J. Schulze. Appl. Phys. Lett., 101, 141110 (2012).

[4] S. Gupta, Y.-Ch. Huang, Y. Kim, E. Sanchez, K.C. Saraswat. IEEE Electron Dev. Lett., 34, 831 (2013).

[5] R. Chen, S. Gupta, Y.-Ch. Huang, Y. Huo, C.W. Rudy, E. Sanchez, Y. Kim, T.I. Kamins, K.C. Saraswat, J.S. Harris. Nano Lett., 14, 37 (2014). 
[6] S. Wirths, D. Stange, M.-A. Pampillón, A.T. Tiedemann, G. Mussler, A. Fox, U. Breuer, B. Baert, E. San Andrés, N.D. Nguyen, J.-M. Hartmann, Z. Ikonic, S. Mantl, D. Buca. ACS Appl. Mater. Interfaces, 7, 62 (2015).

[7] K. Kostecki, M. Oehme, R. Koerner, D. Widmann, M. Gollhofer, S. Bechler, G. Mussler, D. Buca, E. Kasper, J. Schulze. ECS Trans., 64, 811 (2014).

[8] S. Oguz, W. Paul, T.F. Deutsch, B-Y. Tsaur, D.V. Murphy. Appl. Phys. Lett., 43, 848 (1983).

[9] J. Xie, A.V.G. Chizmeshya, J. Tolle, V.R. D’Costa, J. Menendez, J. Kouvetakis. Chem. Mater., 22, 3779 (2010).

[10] S. Wirths, Z. Ikonic, A.T. Tiedemann, B. Hollander, T. Stoica, G. Mussler, U. Breuer, J.M. Hartmann, A. Benedetti, S. Chiussi, D. Grutzmacher, S. Mantl, D. Buca. Appl. Phys. Lett., 103, 192110 (2013).

[11] V. Mashanov, V. Ulyanov, V. Timofeev, A. Nikiforov, O. Pchelyakov, Ing-Song Yu, Henry Cheng. Nanoscale Res. Lett., 6, 85 (2011).

[12] A.E. Dolbak, B.Z. Olshanetsky. Cent. Eur. J. Phys., 6, 634 (2008).

[13] A.I. Nikiforov, V.I. Mashanov, V.A. Timofeev, O.P. Pchelyakov, H.-H. Cheng. Thin Sol. Films, 557, 188 (2014).

[14] A. Harwit, P. Pukite, J. Angilello, S. Iyer. Thin Sol. Films, 184, 395 (1990).

[15] J. Tolle, A. Chizmeshya, Y. Fang, J. Kouvetakis, V. D’Costa, C. Hu, J. Menendez, I. Tsong. Appl. Phys. Lett., 89, 231924 (2006).

[16] R.R. Lieten, J.W. Seo, S. Decoster, A. Vantomme, S. Peters, K.C. Bustillo, E.E. Haller, M. Menghini, J.P. Locquet. Appl. Phys. Lett., 102, 052106 (2013).

[17] M. Oehme, K. Kostecki, M. Schmid, F. Oliveira, E. Kasper, J. Schulze. Thin Sol. Films, 557, 169 (2014).

[18] J. Xie, J. Tolle, V. D'Costa, A. Chizmeshya, J. Menendez, J. Kouvetakis. Appl. Phys. Lett., 95, 181909 (2009).

[19] A.K. Gutakovskii, A.L. Chuvilin, S.A. Song. Bull. Rus. Acad. Sci., ser. 3hys., 71, 1426 (2007).

[20] T. Tsukamoto, N. Hirose, A. Kasamatsu, T. Mimura, T. Matsui, Y. Suda. Appl. Phys. Lett., 106, 052103 (2015).

[21] K. Ueda, K. Kinoshita. Surf. Sci., 145, 261 (1984).

Редактор Г.А. Оганесян

\section{Strained multilayer structures with pseudomorphic GeSiSn layers}

\author{
V.A. Timofeev ${ }^{+}$, A.I. Nikiforov ${ }^{+*}$, A.R. Tuktamyshev ${ }^{+}$, \\ M.Yu. Yesin ${ }^{+}$, V.I. Mashanov ${ }^{+}$, A.K. Gutakovskii ${ }^{+}$, \\ N.A. Baidakova ${ }^{\wedge}$
}

+ Rzhanov Institute of Semiconductor Physics, Siberian Branch of Russian Academy of Sciences, 630090 Novosibirsk, Russia

* National Research Tomsk Polytechnic University, 634050 Tomsk, Russia

$\triangle$ Institute for Physics of Microstructures, Russian Academy of Sciences, 607680 Nizhny Novgorod, Russia

\begin{abstract}
The temperature and composition dependences of the critical thickness of the 2D-3D transition for GeSiSn films on $\mathrm{Si}(100)$ have been studied. First regularities of the formation of multilayer structures containing pseudomorphic GeSiSn layers directly on $\mathrm{Si}$ substrate without the relaxed buffer layers were investigated. The possibility to create multilayer structures on the basis of pseudomorphic GeSiSn layers is shown and parameters of the crystal lattice are defined by the transmission electron microscopy. Structures obtained by the molecular-beam epitaxy have demonstrated the photoluminescence for Sn content in the GeSiSn layers from 3.5 to $5 \%$.
\end{abstract}

